\title{
ПРИМЕНЕНИЕ ИНТЕЛЛЕКТУАЛЬНЫХ ИНФОРМАЦИОННЫХ ТЕХНОЛОГИЙ ДЛЯ ПОВЫШЕНИЯ ЭФФЕКТИВНОСТИ ПРИНЯТИЯ УПРАВЛЕНЧЕСКИХ РЕШЕНИЙ
}

\section{APPLICATION OF INTELLECTUAL INFORMATION TECHNOLOGIES TO INCREASE THE EFFICIENCY OF MANAGEMENT DECISION MAKING}

I. Kharin

Summary. The article discusses the features of the use of intelligent information technologies to improve the efficiency of managerial decision-making. In the course of the research, the prospects and possibilities of using intelligent agents, namely those based on precedents and allowing the search for solutions in the state space, have been analyzed. Recommendations have been developed regarding the construction of metrics for intelligent agents. The article also presents a model of a management decision support system, which is based on the progressive technology of multidimensional conceptual representation.

Keywords: decision making, data mining, information technology, multidimensional representation, model.
$\mathbf{P}$ азработка систем поддержки принятия решений в режиме реального времени всегда было актуальной задачей в сфере автоматизации управленческой деятельности человека. Бурное развитие современного общества детерминировано стремительным увеличением и динамичностью роста разнообразной информации. Эта информация становится все более структурированной и гораздо сложнее поддается четкой формализации, следовательно, так называемые «стандартные» базы данных и системы принятия решений на их основе требуют значительных изменений и развития.

Анализируя опыт практического применения средств автоматизированного управления, информационных и информационно-аналитических систем в комплексах оперативного менеджмента на фоне всевозрастающих объемов информации, необходимой для принятия решений, можно сделать вывод о наличии практической потребности в создании и применении средств автоматизации на основе использования эле-
Харин Илья Андреевич

Национальный исследовательский университет

МЭИ

play22@list.ru

Аннотация. В статье рассмотрены особенности использования интеллектуальных информационных технологий для повышения эффективности принятия управленческих решений. В процессе исследования проанализированы перспективы и возможности применения интеллектуальных агентов, а именно тех, которые основаны на прецедентах и тех, которые позволяют осуществлять поиск решений в пространстве состояний. Кроме того, разработаны рекомендации, касающиеся построения метрик интеллектуальных агентов. Также в статье представлена модель системы поддержки принятия управленских решений, которая базируется на прогрессивной технологии многомерного концептуального представления.

Ключевые слова: принятие решений, интеллектуальный анализ, информационные технологии, многомерное представление, модель.

ментов искусственного интеллекта и инженерии знаний. При этом результатом интеллектуального анализа данных должен быть такой информационный продукт, который позволяет осуществить конкретное управленческое действие без чрезмерного «погружения» лица, принимающего решение, в детали базовых данных или промежуточной аналитики.

Такая практическая задача может быть решена несколькими путями. Во-первых, - это внедрение в архитектуру существующих систем промежуточных аппаратных и программных элементов, которые бы объединяли разрозненные сети в одну вычислительную среду, таким образом реализуя схему сетевого взаимодействия с интеллектуальными компонентами. Во-вторых, - это разработка (использование) нового интегрированного аппаратного и программного обеспечения, которое бы заменило собой существующие традиционные автоматизированные комплексы на качественно новые интеллектуальные системы управления. Кроме того, необходимо акцентировать внимание на том, что, как 
правило, современные системы поддержки принятия решений требуют мультидисциплинарного взаимодействия, которое включает в себя теории баз данных, методы имитационного моделирования, искусственного интеллекта, нейронных сетей, ситуационного анализа и интерактивных компьютерных систем.

Однако, на этапах проектирования и реализации интеллектуальных информационных технологий, для повышения эффективности принятия управленческих решений, существует ряд методологических и технологических проблем. В частности, такие проблемы заключаются в отсутствии концептуальной целостности и согласованности между отдельными приемами и методами инженерии знаний; жесткости разработанных программных средств и их низкой адаптивной способности; использовании в информационных комплексах слабоформализованных знаний в определенной предметной области, а также особенностях реализации логической или содержательной обработки информации.

Таким образом, необходимость решения обозначенных задач, более четкое определение закономерностей формирования и реализации отдельных информационных процессов субъектом управления и обоснованность использования соответствующей информационной технологии, предопределяет выбор темы данной статьи, а также составляют концептуальную основу проводимого исследования.

В общем виде теоретическая проблема построения современных систем поддержки принятия управленческих решений может быть сформулирована как проблема информационной и интеллектуальной интеграции, исследованию которой посвящено большое количество работ отечественных и зарубежных авторов, к числу которых можно отнести: Ягольникова Д.В., Шведун А.А., Гатчина Ю.А., Chen, B.; Ge, Y.H.; Wang, H.; Min, Hokey; Onggo, B. S.

Теоретические и методологические принципы создания и использования интеллектуальных информационных систем и технологий детально описаны Секлетовой Н.Н., Цапаевым М.А., Федоренко С.И., Fernandez, Maria Isabel; Alfred Zimmermann, Rainer Schmidt, Lakhmi C. Jain.

Ряд исследователей занимаются описанием основных модулей и инструментов, которые используются для создания интеллектуальных систем принятия решений (Ивановский М.А., Батурина Е.В., Алмали А.А., Mathirajan, M., Al-Kasasbeh, Maha). Детальному анализу гибридного подхода в проектировании интеллектуальных систем, который позволяет синтезировать каче- ственно различные подходы к обоснованию решений, посвящены труды Шабанова Р.М., Микушина Н.А., Липницкого С.Ф., Alexis Chen, B., Ge, Y.H.

Однако, несмотря на широкий научный интерес к исследуемой тематике, а также тот факт, что интеллектуальные информационные технологии используются уже более полувека, вопросы их применения с целью оптимизации менеджмента и бизнес-процессов компаний остаются открытыми и требуют дополнительной проработки.

В частности, в более углубленном исследовании нуждаются вопросы развития концепций, теоретических положений, моделей и методов построения программного обеспечения, а также наделение указанных систем интеллектуальными свойствами, характерными для современных управленческих комплексов.

Также не в полной мере решенными являются проблемы реализации языковых и инструментальных средств (универсальных и проблемно-ориентированных), которые поддерживают полный жизненный цикл разработки управленческих решений. Особого внимания заслуживает разработка обобщающих формализмов для различных классов моделей и методов поддержки принятия решений, базирующихся на интеллектуальных информационных технологиях.

Итак, принимая во внимание вышеизложенное, цель статьи заключается в детальном изучении особенностей использования интеллектуальных информационных технологий для повышения эффективности принятия управленческих решений.

Интеллектуальные информационные технологии представляют собой информационные технологии, которые помогают менеджеру ускорить синтез управленческих решений [2]. В своем развитии информационные технологии, применяемые в системе поддержки принятия решений прошли следующие этапы: разработка баз данных (DB - Data Base), технология аналитической обработки данных в режиме онлайн (OLAP - On-line Analytical Processing), технология интеллектуального анализа данных (DM - Data Mining), системы поддержки выполнения решений (EPSS - Electronic Performance Support Systems), мультиагентные системы (MA).

Современный уровень развития интеллектуальных информационных технологий, которые используются для построения систем поддержки принятия управленческих решений, происходит в двух направлениях разработки интеллектуальных агентов (ИА):

- ИА, основанные на прецедентах (Case-Based Reasoning (CBR); 


\section{Пользователь}

$\downarrow$

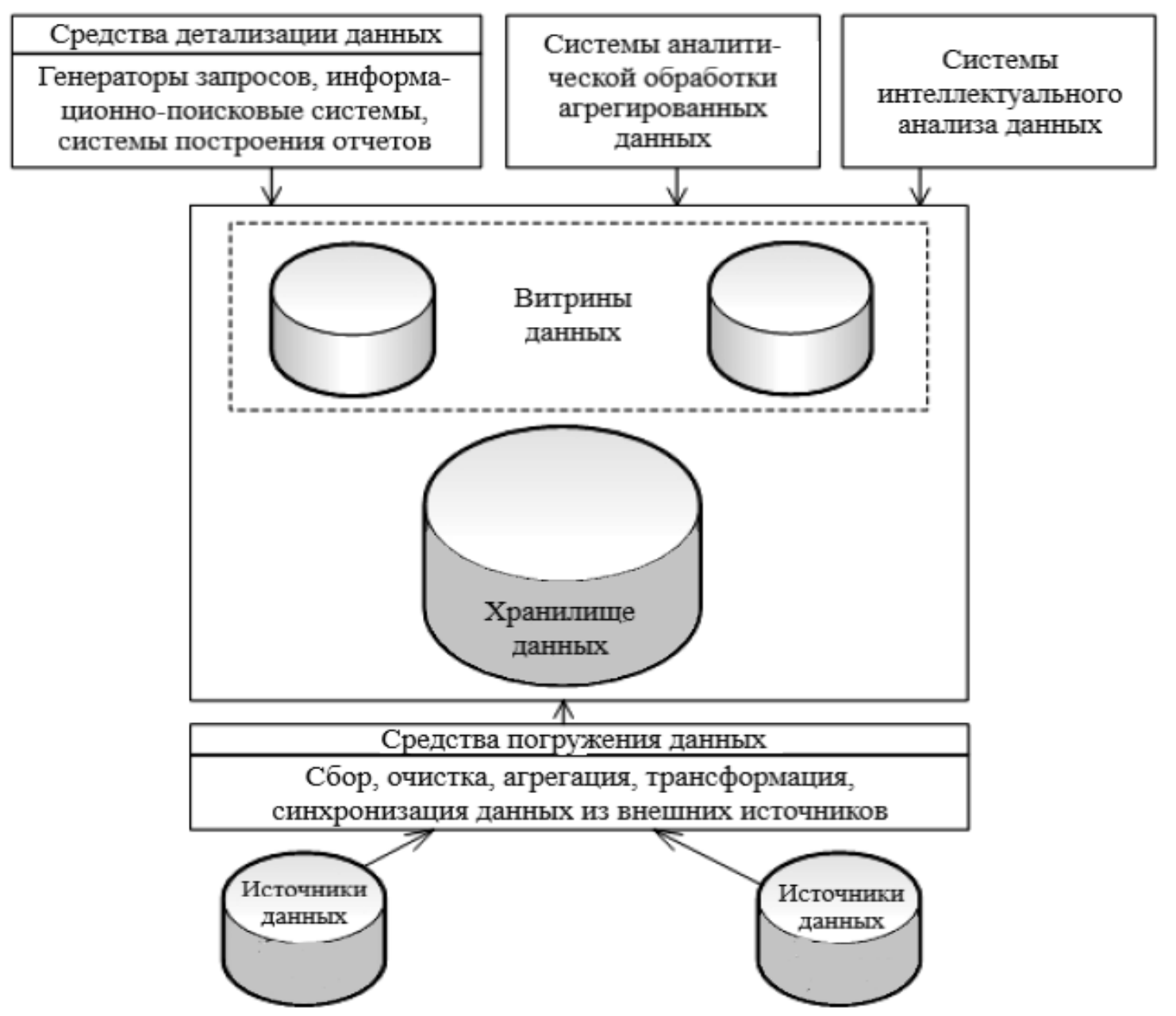

Рис. 1. Модель системы поддержки принятия управленских решений на основе многомерного концептуального представления

- ИА планирования деятельности (поиск в пространстве состояний).

Выбор ИА зависит от задачи, которую необходимо решить управленцу. Метод вывода по прецедентам эффективен, когда основным источником знаний о задаче является опыт, а не теория; потенциальные решения не уникальны для конкретной ситуации, а могут быть использованы в других случаях; целью решения задачи является получить не гарантировано верное решение, а наилучший вариант из всех возможных. Интеллектуальная информационная технология, которая основана на прецедентах, является методом выработки, построения управленческого решения по результатам поиска аналогий, хранящихся в базе прецедентов. Такой прецедент называют релевантным.
С математической точки зрения среди элементов множества прецедентов $P_{r}=\left\{P_{r l}, P_{r 2}, \ldots, P_{r N}\right\}$ релевантным $P_{r k}$ является прецедент, для которого расстояние до текущей проблемной ситуации $S$ является минимальным, то есть:

$$
P_{r k}=\arg \min d\left(P_{r i}, S\right)
$$

Суть и особенности ИА планирования деятельности заключаются в том, что они предполагают достижение целевого состояния. Прежде всего, ИА должен построить план достижения этого состояния со всеми возможными альтернативами.

Процесс планирования основывается на декомпозиции. Задача планирования $Z P$ содержит 3 составля- 
ющих: множество состояний $S t$, множество действий $A$, множество целевых состояний Goal (состояний цели). Все это описывается следующим выражением:

$$
Z P=\langle S t, A, \text { Goal }\rangle
$$

Итак, для планирования деятельности ИА должен уметь оценивать состояния и действия.

Очевидно, что для обоих классов рассматриваемых систем необходима метрика. В первом случае для оценки релевантности прецедентов, во втором случае - для оценки релевантности состояний. От способа определения этой метрики напрямую зависит эффективность работы ИА и соответственно оптимальность принимаемого управленческого решения.

По мнению автора, такой способ должен базироваться на четком и аргументированном стандарте баз знаний. В области инженерии знаний таким стандартом являются онтологии. Поэтому представляется целесообразным для построения метрики использовать онтологии.

Под моделью онтологии $O$ будем понимают тройку следующего вида:

$$
O=\langle C, R, F\rangle
$$

где $C$ - понятие, $R$ - отношение между понятиями, $F$ - интерпретация понятий и отношений (аксиомы). Аксиомы устанавливают семантические ограничения для системы понятий и отношений.
Еще одной инновационной информационной интеллектуальной технологией, которая имеет значительный нереализованный потенциал и широкие возможности использования в будущем в системах принятия решений, является многомерное концептуальное представление (multidimensional conceptual view), которое представляет собой множественную перспективу, состоящую из нескольких независимых измерений, в рамках которых могут быть проанализированы определенные совокупности данных. Каждое измерение охватывает направления консолидации данных, состоящие из серии последовательных уровней обобщения, где каждый высший уровень соответствует большей степени агрегации данных.

На рис. 1 представлена модель интеллектуальной системы поддержки принятия решений на базе многомерного концептуального представления. Ее составляющими элементами являются: информационное хранилище данных, средства погружения данных, средства детализации данных, системы аналитической обработки агрегированных данных, системы интеллектуального анализа данных.

Таким образом, резюмируя полученные результаты, можно отметить, что использование интеллектуальных информационных технологий для поддержки принятия решений способно повысить эффективность и качество решения управленческих задач за счет совершенствования информационного обеспечения, снижения возможных ошибок и более комплексной обработки данных.

\section{ЛИТЕРАТУРА}

1. Секлетова Н.Н., Цапаев М.А. Постановка задач при проектировании информационной системы для поддержки принятия решений // Экономика и социум. 2019. № 1-1 (56). С. 1012-1014.

2. Architecting the digital transformation: digital business, technology, decision support, management / Alfred Zimmermann, Rainer Schmidt, Lakhmi C. Jain. Cham, Switzerland: Springer, 2021. 243 p.

3. Essentials of management information systems / Kenneth C. Laudon, Jane P. Laudon. Harlow, United Kingdom: Pearson Education Limited, 2019. 273 p.

( Харин Илья Андреевич ( play22@list.ru)

Журнал «Современная наука: актуальные проблемы теории и практики» 